\title{
Technical and clinical analysis of microEEG: a miniature wireless EEG device designed to record high-quality EEG in the emergency department
}

\author{
Ahmet Omurtag', Samah G Abdel Baki', Geetha Chari², Roger Q Cracco $^{3}$, Shahriar Zehtabchi', \\ André A Fenton ${ }^{5}$ and Arthur C Grant ${ }^{6 *}$
}

\begin{abstract}
Background: We describe and characterize the performance of microEEG compared to that of a commercially available and widely used clinical EEG machine. microEEG is a portable, battery-operated, wireless EEG device, developed by Bio-Signal Group to overcome the obstacles to routine use of EEG in emergency departments (EDs).

Methods: The microEEG was used to obtain EEGs from healthy volunteers in the EEG laboratory and ED. The standard system was used to obtain EEGs from healthy volunteers in the EEG laboratory, and studies recorded from patients in the ED or ICU were also used for comparison. In one experiment, a signal splitter was used to record simultaneous microEEG and standard EEG from the same electrodes.

Results: EEG signal analysis techniques indicated good agreement between microEEG and the standard system in 66 EEGs recorded in the EEG laboratory and the ED. In the simultaneous recording the microEEG and standard system signals differed only in a smaller amount of $60 \mathrm{~Hz}$ noise in the microEEG signal. In a blinded review by a board-certified clinical neurophysiologist, differences in technical quality or interpretability were insignificant between standard recordings in the EEG laboratory and microEEG recordings from standard or electrode cap electrodes in the ED or EEG laboratory. The microEEG data recording characteristics such as analog-to-digital conversion resolution (16 bits), input impedance $(>100 \mathrm{M} \Omega)$, and common-mode rejection ratio $(85 \mathrm{~dB})$ are similar to those of commercially available systems, although the microEEG is many times smaller ( $88 \mathrm{~g}$ and $9.4 \times 4.4 \times 3.8 \mathrm{~cm}$ ).

Conclusions: Our results suggest that the technical qualities of microEEG are non-inferior to a standard commercially available EEG recording device. EEG in the ED is an unmet medical need due to space and time constraints, high levels of ambient electrical noise, and the cost of 24/7 EEG technologist availability. This study suggests that using microEEG with an electrode cap that can be applied easily and quickly can surmount these obstacles without compromising technical quality.
\end{abstract}

Keywords: Electroencephalography (EEG), EEG technology, EEG machine, Signal analysis, Emergency department

\section{Background}

Obtaining rapid EEGs in the ED could improve patient care by narrowing the differential diagnosis and avoiding unnecessary tests, procedures, admissions, and costs. Approximately two to ten percent of all patients presenting to US emergency departments (EDs) present with altered mental status (AMS), with the most

\footnotetext{
* Correspondence: arthur.grant@downstate.edu

${ }^{6}$ Departments of Neurology, and Physiology \& Pharmacology, State University of New York, Downstate Medical Center, Brooklyn, USA Full list of author information is available at the end of the article
}

frequent underlying cause being neurological disease [1]. Studies show that ED patients with AMS whose initial evaluation includes EEG are diagnosed more accurately and sooner than those without an EEG [2-9]. Despite its utility, routine use of EEG in the ED faces numerous obstacles. Hospital EEG laboratories are rarely open around the clock [10,11]. An informal Internet-based survey found only $2 \%$ of EDs are equipped with EEG machines or have a technologist who can properly apply EEG electrodes, troubleshoot problems, and record a technically adequate study. 
Attaching a full set of EEG electrodes can take up to $30 \mathrm{~min}$ and even longer with an uncooperative or agitated patient. The long wires leading from the electrodes to the traditional EEG machine act as antennas and often pick up relatively high-voltage ambient electrical noise because of the large number of noise sources in the ED environment. The electrode wires may also constrain movement and limit access of medical personnel to the patient in the typically cramped emergency department setting. Other reasons for the infrequent use of EEG in the ED include lack of space, cost of EEG machines, and the difficulty of finding skilled EEG interpreters available 24/7 [12].
This article describes a new EEG device ("microEEG") that can potentially overcome these limitations (Figure 1). microEEG is a miniature, portable, battery-powered, and wireless EEG device. Although each of these qualities is not in itself unique, their combination in a single device that can record high-quality EEG signals from high and unbalanced impedances sets microEEG apart from other available wireless EEG machines. microEEG was developed by Bio-Signal Group, optimized for obtaining highquality EEG recordings in the ED, and has been certified to meet electromagnetic compatibility (EMC) and medical safety standards. Since the initial review of this article, the microEEG device has received FDA 510(k)

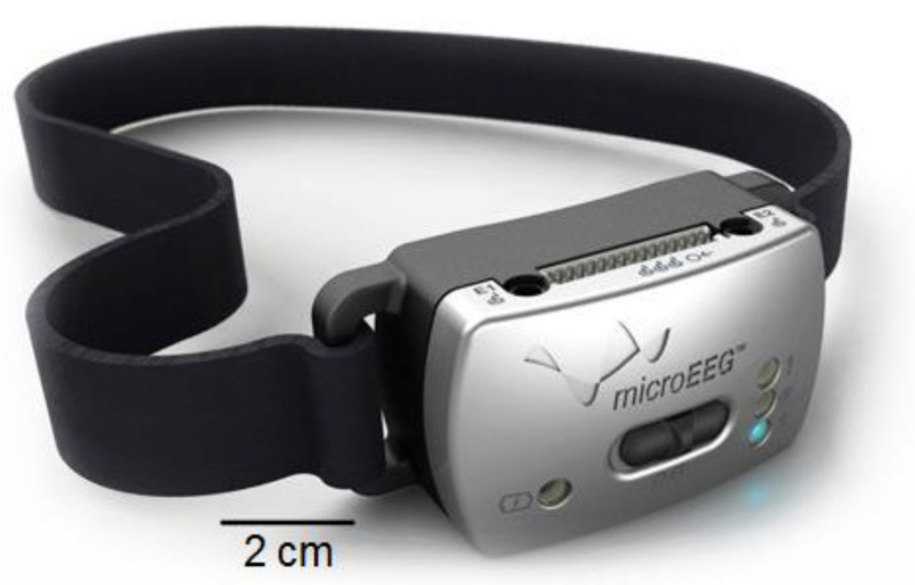

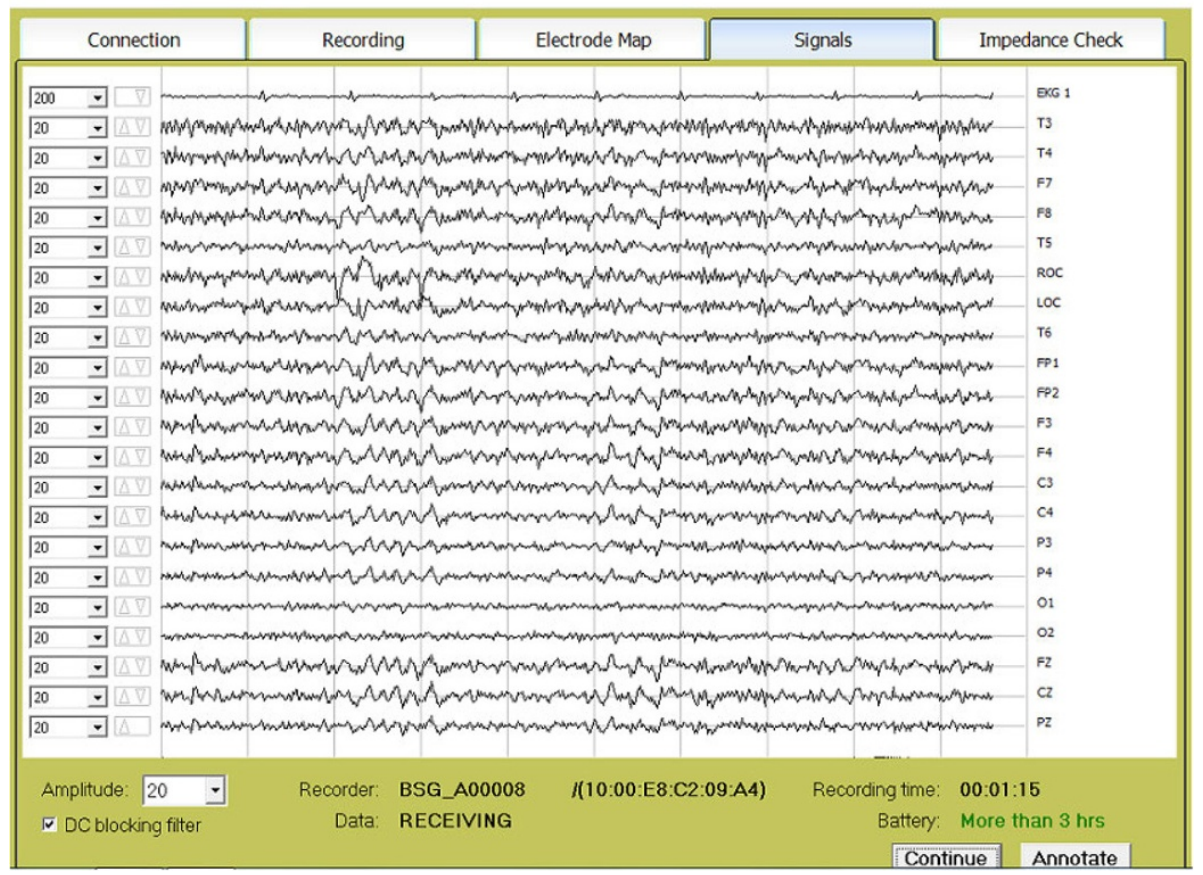

Figure 1 The microEEG system. The recorder and transmitter (top) and the interface of the software running on a PC that allows the user to control the microEEG, view the signals, adjust the display scale and filters, check the battery, and enter annotations (bottom). 
approval. The aims of this study were to evaluate both the feasibility of the microEEG for routine use in EDs and the quality of its signals relative to those acquired by a standard, commercially available EEG machine.

\section{Methods}

The microEEG, at merely $9.4 \times 4.4 \times 3.8 \mathrm{~cm}$ and $88 \mathrm{~g}$, is about the size and weight of a cellular telephone and can work equally well with both standard cup electrodes and readily available and rapidly deployable electrode caps, whose electrode-scalp impedances are relatively high. The microEEG digitizes the EEG signals close to the electrodes, transmits the digital data wirelessly to a personal computer located within $10 \mathrm{~m}$, and stores the data on an on-board memory card. Custom software running on the PC controls the device, measures electrode impedances (including ground and reference electrodes), displays the signals and impedances, allows the entry of annotations, and writes data to the hard disk. The software can also stream data to a remote server where an authorized user can review and interpret the EEG.

Table 1 shows the manufacturer's specifications for the microEEG, Nicolet Monitor (which we use as the comparison or "standard" system), and Trackit, another portable system that we identified as the commercially available device with the closest technical specifications to the microEEG.

Four experiments were performed to compare microEEG recordings to those of the standard system, with the EEGs obtained in various clinical environments. Comparisons were made of both technical data and clinical interpretations rendered by a blinded reviewer. The

Table 1 Properties of selected EEG devices

\begin{tabular}{|c|c|c|c|}
\hline Product & microEEG & Nicolet Monitor & $\begin{array}{l}\text { Trackit } \\
\text { Recorder }\end{array}$ \\
\hline Company & Bio-Signal Group & CareFusion Corp. & Lifelines Ltd. \\
\hline $\begin{array}{l}\text { A/D Converter } \\
\text { resolution (bits) }\end{array}$ & 16 & 16 & 16 \\
\hline $\begin{array}{l}\text { Voltage resolution } \\
(\mu \mathrm{V})\end{array}$ & 0.15 & 0.15 & 0.15 \\
\hline $\begin{array}{l}\text { Maximum input } \\
\text { range }\end{array}$ & 10 mV p.to.p. & 10 mV p.to.p. & 10 mV p.to.p. \\
\hline Sampling rate & $1,000 \mathrm{~Hz}$ & Up to $2,000 \mathrm{~Hz}$ & Up to $256 \mathrm{~Hz}$ \\
\hline Bandwidth & $0.15-500 \mathrm{~Hz}$ & $0.16-1,000 \mathrm{~Hz}$ & $0.16-128 \mathrm{~Hz}$ \\
\hline $\begin{array}{l}\text { Input impedance } \\
(\mathrm{M} \Omega)\end{array}$ & $>100$ & $>100$ & $>100$ \\
\hline $\begin{array}{l}\text { Separately test } \\
\text { GND/REF } \\
\text { impedance }\end{array}$ & Yes & No & No \\
\hline $\begin{array}{l}\text { Number of } \\
\text { channels }\end{array}$ & 32 & 64 & $\begin{array}{l}\text { 12-36 } \\
\text { (depending on } \\
\text { model) }\end{array}$ \\
\hline
\end{tabular}

four experiments and the techniques used in the technical comparisons are described in the subsequent five subsections. See the Appendix for additional technical details. The studies were approved by the SUNY Downstate Medical Center IRB, and written informed consent was obtained from all volunteer subjects.

\section{Parallel recording}

A parallel recording was made simultaneously with the microEEG and the standard system, with gold-plated cup electrodes on a healthy adult volunteer subject in the EEG laboratory. This permitted direct comparison of signals between the devices without any possibility of confounding from differences in signal origin. A signal splitter bifurcated the signal from each cup electrode into two identical streams. Prior to the use of the splitter in the EEG recordings, its accuracy was verified with standard engineering techniques, which included delivering currents of varying frequency and amplitude to the electrode leads through a signal generator and comparing the resulting signal in the two streams. Study duration was $15 \mathrm{~min}$.

\section{microEEG in the EEG Laboratory}

Fifteen 20-30-min microEEG recordings were obtained on adult volunteers. Signal properties of these recordings were compared to those of 28 standard EEGs. Time and frequency domain properties of the microEEG data were compared to those of the standard system with all EEGs recorded in the EEG laboratory. The purpose of this experiment was to test the microEEG as a signal acquisition system without changing electrodes or the environment of a typical EEG examination. All EEGs were recorded using standard 9-mm gold-plated cup electrodes placed according to the international 10-20 system. The subject's scalp was rubbed with NuPrep EEG skin prepping gel, and the electrodes were attached using Ten20 conductive paste.

\section{microEEG in the ED}

In the next experiment, 51 microEEG recordings obtained in the ED were compared to standard recordings obtained in the ED and ICU. microEEG recordings were made with the rapidly deployable and commercially available Electro-Cap (23 studies) and with standard gold-plated cup electrodes (28 studies). The Electro-Cap is made of an elastic, spandex-type fabric with recessed, pure tin electrodes attached to the fabric. A small amount of electro-gel was injected through a hole in the center of each electrode to minimize the electrode-scalp impedance. For a realistic clinical simulation of the ED environment, volunteers were connected to noninvasive devices including a pulse oximeter, EKG machine, and oxygen nasal mask. A saline drip was connected to the 
subject by taping a blunt needle to his or her arm. Blood pressure (BP) monitoring was done by an automated pressure cuff placed around the subject's arm for BP detection every $10 \mathrm{~min}$ during the EEG recording. Electrode impedances were measured at the beginning and end of each recording. Standard recordings consisted of 7 ED studies and 11 ICU studies randomly selected from all of the ED and ICU studies obtained in the prior 1 year at SUNY Downstate Medical Center.

\section{Assessment by a clinical neurophysiologist}

A board-certified clinical neurophysiologist reviewed 37 de-identified 30-min EEGs obtained with either the microEEG or the standard system. The data set consisted of 14 microEEG studies recorded in the ED with the Electro-Cap, 13 microEEG studies recorded in the ED with cup electrodes, 8 standard EEGs recorded in the EEG laboratory, and 2 standard EEGs recorded in the ICU. All recordings were reviewed using Insight II, Persyst Development Corp. (Prescott, AZ). The reviewer determined whether each recording was technically adequate for clinical interpretation, i.e., was not substantially obscured by artifacts that rendered the study uninterpretable for clinical purposes.

\section{Measures of agreement}

The simultaneous parallel recording with the microEEG and the standard system provided a unique opportunity to compare the recorded signals in the time domain. We computed the short time correlations between the two signals and examined their values throughout the recording on all channels. The correlations were also used as a guide to focus visual inspection on specific segments of the recording. Standard deviations, higher order statistics, and Hjorth mobility and complexity parameters [13-15] for each system's signals were also examined.

Frequency domain measures were used to compare microEEG and standard system recordings in all of the experiments. We began by computing the power spectral density (PSD) of each channel over a 500-s interval. Spectral properties were derived from the PSD data.

Combinations of such indicators have been used with varying levels of success to detect both normal and abnormal EEG findings, as well as artifacts. Examples include detection of rhythmic discharges in newborns [16], multi-morphologic ictal patterns in the human longterm EEG [17], muscle and electrical noise artifacts [18], seizure prediction (reviewed by [19]), early patientspecific seizure detection [20], classification of sleep stages [21,22], and identification of resting state [23] or epileptic [24] brain networks.

When sampling rates for the microEEG and standard system were unequal, the microEEG signal was resampled to the time points of the standard system using cubic spline interpolation [25]. Whenever needed (as indicated in the Appendix), the data were bandpass filtered with zero phase shift by a sixth order digital Butterworth filter. This diverse set of measures from both the time and frequency domains provided a comprehensive measure of technical performance.

\section{Results}

Visual inspection of the parallel recordings with an AP bipolar longitudinal montage did not reveal any clinically significant difference between the studies. Figure 2 shows a section of the parallel recordings displayed with Insight II (Persyst Corp.). Low-pass and notch filters are off, and the resolution is set to high. The microEEG and standard EEG appear nearly identical, although there is greater high frequency noise in the standard EEG (Figure 2, bottom panel). Visual inspection of the remaining microEEG studies revealed that none of the signals contained unexplained artifacts or expected artifacts (e.g., due to muscle, movement, EKG) at levels greater than those found in the standard recordings.

\section{Parallel recording}

Figure 3 illustrates the superimposition of $0.7 \mathrm{~s}$ of simultaneous signal recorded from the microEEG and the standard system at electrodes T6 and O1. These examples were selected to show segments with low $(r \approx 0.5)$ and high $(r \approx 1)$ correlation. The T6 segment illustrates suppression of $60-\mathrm{Hz}$ noise by microEEG. Close examination of all 20 channels throughout the recordings revealed that imperfect correlation was due primarily to relatively greater $60-\mathrm{Hz}$ noise in the standard system.

Table 2 summarizes the statistics of correlation for the entire recording over all electrodes. As expected, correlation was proportional to the amplitude of EEG signals and inversely proportional to $60-\mathrm{Hz}$ noise. For example, the average correlation for channel $\mathrm{O} 2$ during an eyesclosed segment of the EEG (when there is relatively high posterior alpha activity) was $0.960 \pm 0.047$ (mean \pm standard deviation).

Table 3 illustrates the sensitivity of time domain parameters to high-frequency noise. Again, the data were obtained from analyzing the signal on all electrodes throughout the recording. In particular, Hjorth mobility was higher in the standard EEG than microEEG and highly sensitive to high frequency noise. This sensitivity is expected since it is interpretable as the standard deviation of the PSD [14]. By contrast, higher order statistics and Hjorth complexity were unchanged when $60-\mathrm{Hz}$ noise was removed from the signals. The skewness was not significantly different from zero and is not shown. Note that we have defined kurtosis as the fourth central 


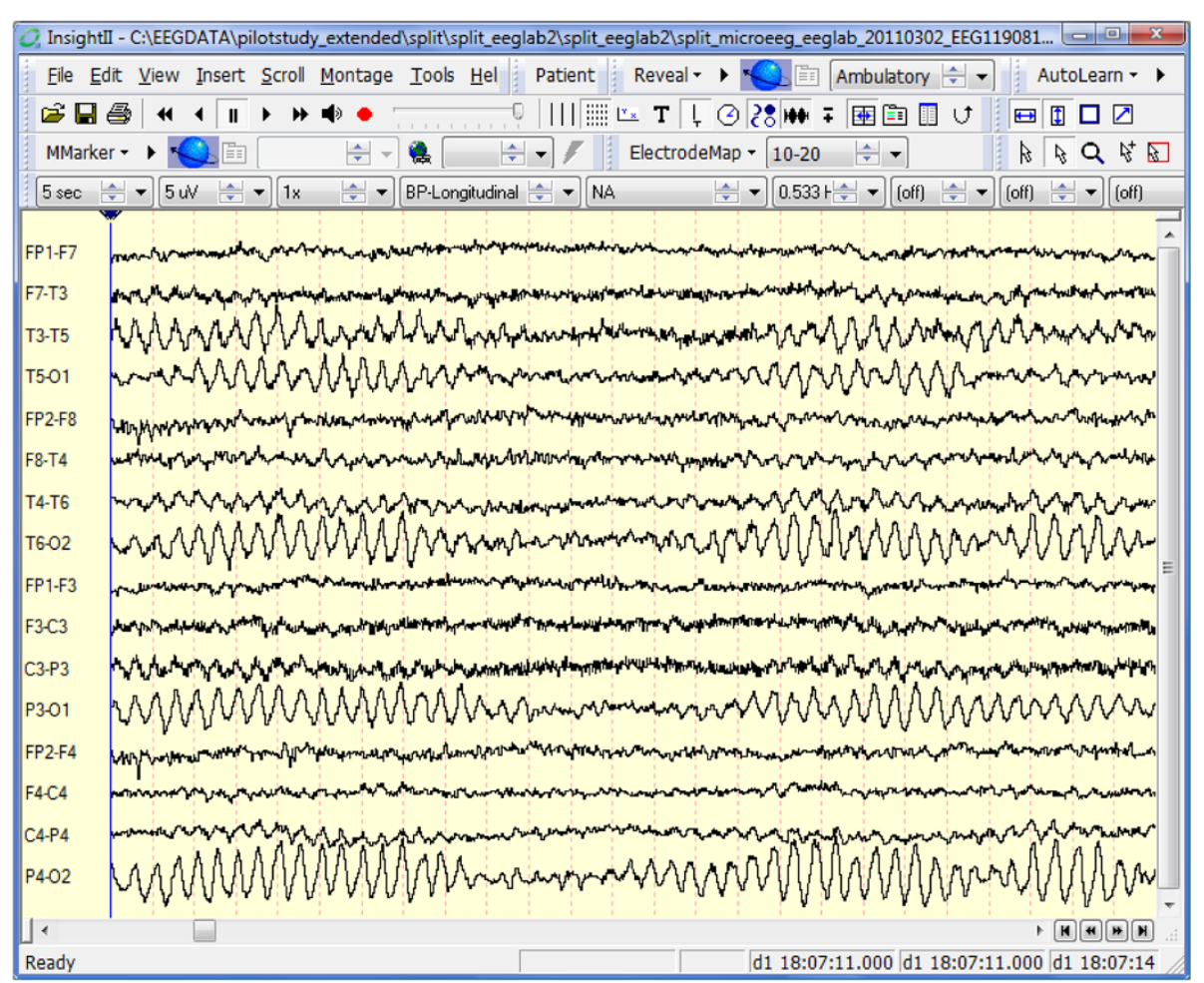

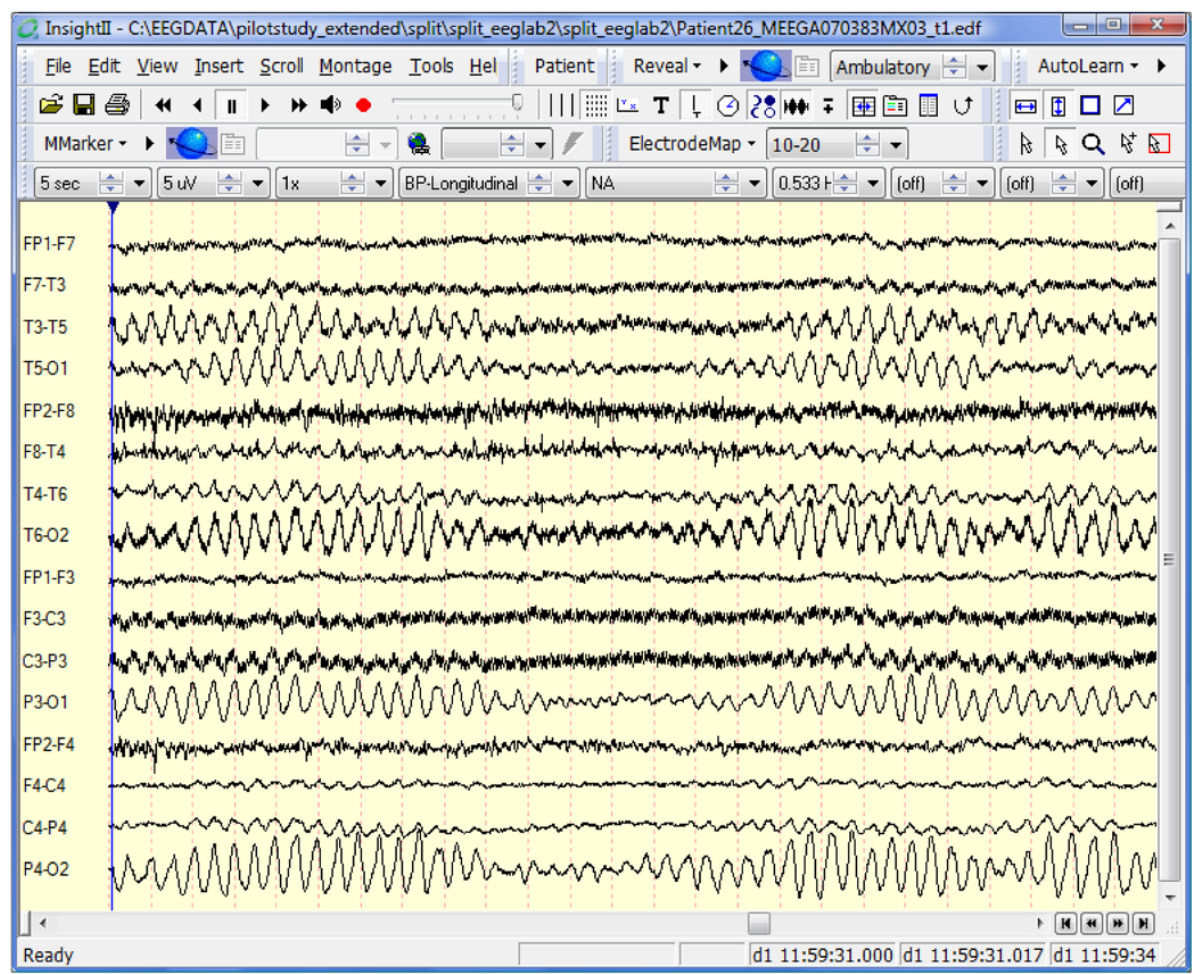

Figure 2 Typical segment from the EEG signals recorded by microEEG (top) in parallel with the standard system (bottom) shown in a standard EEG viewing environment (Insight II from Persyst Development Corp.). Insight's high-pass filter is on, and the resolution is set to high. Note that time and amplitude scales as shown in the viewer controls above the EEG traces are, respectively, $5 \mathrm{~s}$ and $5 \mu \mathrm{V}$. 


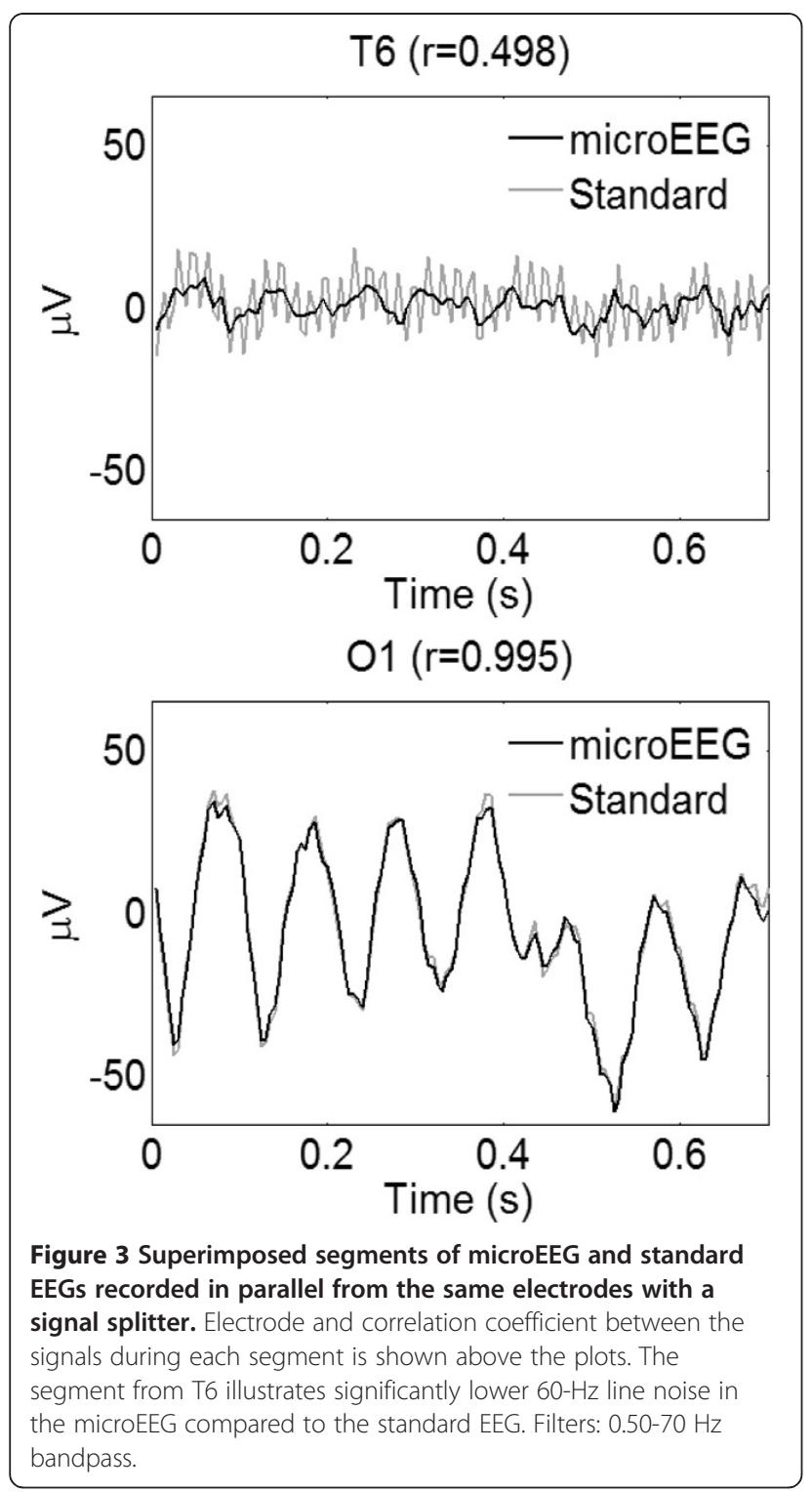

moment of the signal divided by the square of the variance so that kurtosis equals 3 for a normal distribution.

Figure 4 shows the PSD of the signal recorded by the microEEG and standard system on two channels, T6 and O2. These channels contained a significant difference in $10-\mathrm{Hz}$ activity and were selected to illustrate this difference. The curves are smoothed for better visibility except in the neighborhood of $60 \mathrm{~Hz}$. Inspection of the PSD for all channels showed that the standard signal's

Table 2 Averaged short time correlations

\begin{tabular}{llll}
\hline Notch filter & Overall & Eyes open & Eyes closed \\
\hline Off & $0.860 \pm 0.106$ & $0.849 \pm 0.109$ & $0.877 \pm 0.099$ \\
\hline On & $0.911 \pm 0.070$ & $0.902 \pm 0.076$ & $0.926 \pm 0.058$ \\
\hline
\end{tabular}

Correlations between themicroEEG and standard EEG computed for 1-s windows and averaged over the recording for all electrodes. Filters: $0.5-70 \mathrm{~Hz}$ bandpass.
Table 3 Time and frequency domain properties

\begin{tabular}{|c|c|c|c|c|c|c|c|}
\hline & \multicolumn{4}{|c|}{ Time domain } & \multicolumn{3}{|c|}{ Spectral } \\
\hline & $\begin{array}{c}\text { Stdev } \\
(\mu \mathrm{V})\end{array}$ & Kurt & $\begin{array}{c}\text { Mob } \\
(\mathrm{Hz})\end{array}$ & Comp & $\begin{array}{l}\text { MF } \\
(\mathrm{Hz})\end{array}$ & $\begin{array}{c}\text { SEF75 } \\
(\mathrm{Hz})\end{array}$ & SE \\
\hline mEEG (Notch off) & 67.9 & 9.1 & 108.5 & 2.9 & 9.3 & 9.4 & 11.8 \\
\hline STD (Notch off) & 74.7 & 6.9 & 149.4 & 2.3 & 15.6 & 29.0 & 11.4 \\
\hline mEEG (Notch on) & 65.6 & 10.0 & 69.0 & 3.5 & 6.0 & 7.9 & 11.9 \\
\hline STD (Notch on) & 67.9 & 8.9 & 71.8 & 3.2 & 6.8 & 9.4 & 12.0 \\
\hline
\end{tabular}

microEEG and standard EEG recorded in parallel. Filters $0.5-70-\mathrm{Hz}$ bandpass. Data shown are averages over all channels. mEEG, microEEG; STD, standard; Stdev, standard deviation; Kurt, kurtosis; Mob, mobility; Comp, complexity; $M F$, mean frequency; SE, spectral entropy. Kurtosis, mobility, complexity, and spectral entropy are dimensionless.

spectrum agreed well with the microEEG spectrum and was within the confidence limits in all channels and frequencies. Quantities derived from the PSD also had a range of sensitivities to high frequency noise as demonstrated by the results in Table 3. Note that notch filtering both signals drastically reduced the difference in SEF75 and resulted in good agreement.

\section{microEEG in the EEG Laboratory}

Comparison of the band power of the microEEG and standard system indicated that there was good agreement in all frequency ranges except in the lowest range,
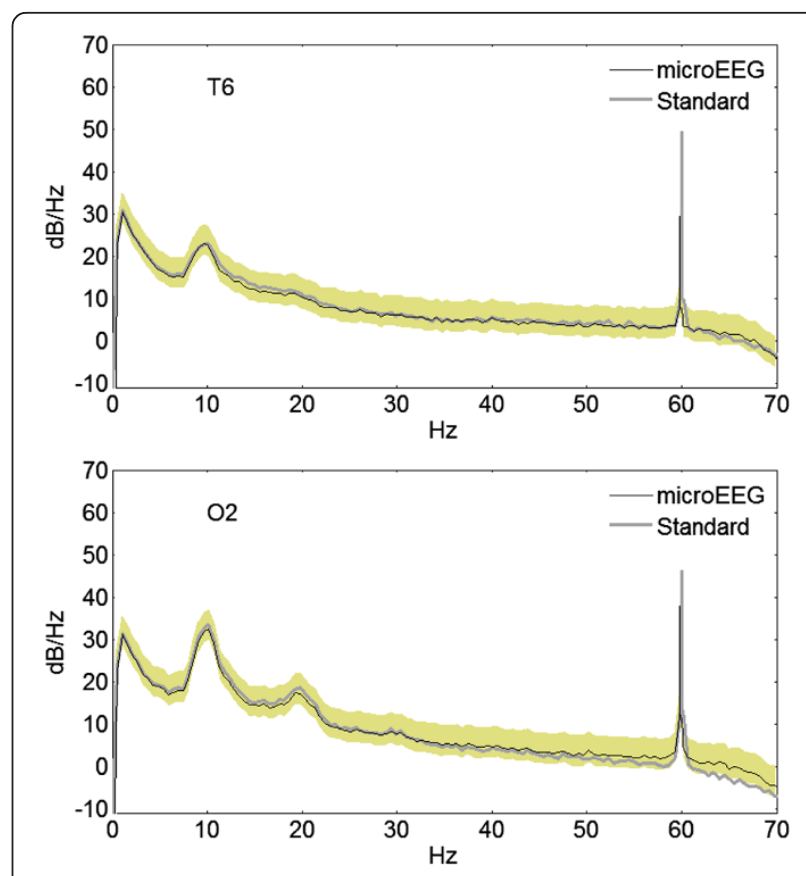

Figure 4 Examples of the power spectral density of microEEG and standard EEG signals recorded in parallel. The curves were smoothed except near $60 \mathrm{~Hz}$. The shaded zone represents the 95\% confidence range for the microEEG signal (the $95 \%$ confidence range for the standard EEG was essentially the same and is not shown). Filters: 0.50-70- $\mathrm{Hz}$ bandpass. 
$0-4 \mathrm{~Hz}$, and at $60 \mathrm{~Hz}$. The mean band power of each channel averaged across recordings was also examined and found to have a similar agreement between the two devices. In the ranges alpha-beta4, the two systems' band powers were nearly equal, while in the theta and beta5 ranges they were within one standard deviation of each other. The standard deviations were calculated from the variability across recordings and channels. Lower power in the $0-4 \mathrm{~Hz}$ band in the microEEG recordings was due to the difference in the hardware high-pass filters: the low frequency cutoff in the microEEG was set at $1 \mathrm{~Hz}$ in these experiments compared to $0.16 \mathrm{~Hz}$ in the standard device. Lower $60-\mathrm{Hz}$ noise in the microEEG compared to the standard system likely resulted from the shorter EEG electrode cable lengths as the common mode rejection ratio of the two systems is the same. This experiment demonstrated that microEEG signals do not contain activity at levels that are unexpectedly different in any frequency range from those recorded by the standard device.

\section{microEEG in the ED}

Figure 5 shows that there was good agreement between the microEEG and the standard system in all frequency bands except at $60 \mathrm{~Hz}$, where the microEEG power was less. The agreement at $0-4 \mathrm{~Hz}$ is much better than in the recordings from the EEG laboratory because the microEEG hardware high-pass cutoff was set to $0.15 \mathrm{~Hz}$. The microEEG recordings with the cup electrodes had results (not shown) similar to those with the ElectroCap with the exception of somewhat higher power near $60 \mathrm{~Hz}$. The mean impedance of Electro-Cap electrodes was $15.9 \pm 17.6 \mathrm{k} \Omega$ with median $8 \mathrm{k} \Omega$ and range 0.9 to $80 \mathrm{k} \Omega$. The mean impedance of cup electrodes was $5.3 \pm 4.2 \mathrm{k} \Omega$ with median $4.3 \mathrm{k} \Omega$ and range 1.2 to 33.3 $\mathrm{k} \Omega$. The impedance of each channel is taken as the

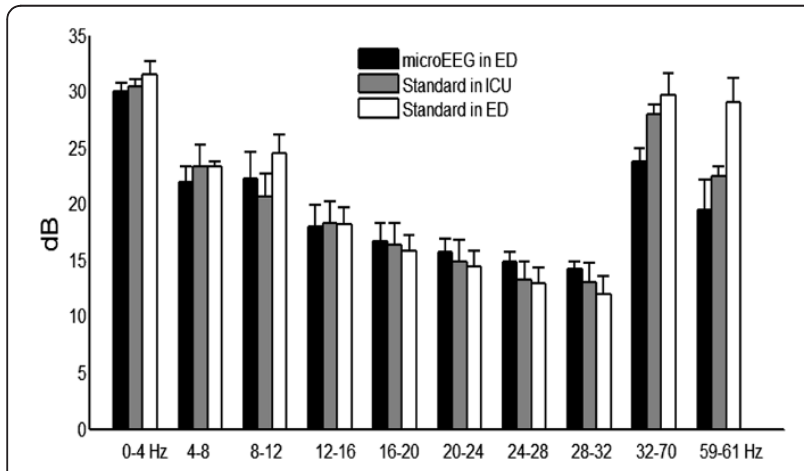

Figure 5 Band power for the microEEG and standard system. microEEG with Electro-Cap in the ED (28 recordings), standard system in the ICU (11 recordings), and standard system in the ED (7 recordings). Error bars indicate the standard deviation of variability due to differences among channels and recordings. Filters $0.5-70 \mathrm{~Hz}$ bandpass. mean of the impedance at the start and end of each recording. The inter-recording variability of impedance was greater than the intra-recording variability. For each recording, the value of the impedance at the end of a recording was strongly correlated to the value at the start $[r=0.84$ (Electro-Cap) and 0.83 (cup electrodes)]. We note that good agreement between the microEEG in the ED and the standard EEG was achieved even though the electrode cap electrode impedances were generally substantially greater than the $<5 \mathrm{k} \Omega$ impedance IFCN standard for digital EEG machines [26].

\section{Assessment by a clinical neurophysiologist}

Two board-certified clinical neurophysiologists independently evaluated the technical quality of ten randomly selected microEEG recordings done in the ED and described in this article (equally divided between cup electrodes and Electro-Cap); they found all of them suitable for making clinically significant interpretations. We then designed the blinded study described in Methods. The blinded reviewer was asked, "Is the recording clinically acceptable?" The results show that the microEEG in the ED performed better than the standard system in the ICU but not as well as the standard system in the EEG laboratory (Table 4). The percentage of Yes answers for the microEEG was significantly lower than for the standard EEG from the EEG laboratory $(z=4.6$, $p<0.05$ test of proportions) but not different from the standard EEG if the EEG laboratory and ICU recordings were not distinguished $(\mathrm{z}=1.21, p>0.05$ test of proportions). Reasons given for clinically unacceptable recordings included wavering baseline, sharply contoured artifacts especially in T5 and T6, excessive EKG artifacts, $60-\mathrm{Hz}$ artifacts, bursts of diffuse artifacts, and muscle artifacts on all leads.

\section{Discussion}

Over the past 2 decades, EEG technology has improved dramatically. Large analog machines with paper recordings have been replaced by much smaller, computer-based digital machines, with all the associated advantages of digital recording and data storage. Preamplifier input impedances have risen without sacrificing CMRR. Despite these advances, recording a

Table 4 Responses given to the question "Is the recording clinically acceptable?"

\begin{tabular}{llll}
\hline & Yes (\%) & Maybe (\%) & No (\%) \\
\hline microEEG/E-Cap/ED & 60 & 27 & 13 \\
microEEG/Cup/ED & 58 & 34 & 8 \\
Standard/EEG lab & 88 & 12 & 0 \\
Standard/ICU & 0 & 50 & 50 \\
\hline
\end{tabular}

Blinded evaluation by a neurologist of a set of recordings that contained both microEEG and standard recordings. 
technically acceptable EEG in electrically hostile environments such as the ED remains a challenge, especially with uncooperative patients in the cramped confines of a busy ED. The most significant challenges are the following: (1) high line noise $(60 \mathrm{~Hz})$ in the recorded signals due to high ambient noise levels, long electrode wires, and relatively high electrode impedances and inter-electrode impedance differences; (2) time needed to attach a full set of EEG electrodes and achieve low electrode-scalp impedances; (3) around the clock availability of trained EEG technologists; and (4) limiting physical access to the patient with the electrode wires and EEG equipment.

This study demonstrates that the microEEG can overcome all of these obstacles. Its miniature size, a built-in rechargeable battery power source, and wireless transmission of digitized EEG data eliminate the physical access problems and space requirements associated with EEG electrode wires, recording equipment, and power cables. The engineering specifications of the microEEG (e.g., A/D converter resolution, sampling rate, input impedance, CMRR, number of channels, etc.) are comparable to currently available commercial systems. However, because the microEEG is wireless and small enough to be rigidly attached to the patient, for example on the head using a headband or on an electrode cap, it can be implemented with very short electrode wires. The short cables, combined with the on-board DC power source, resulted in microEEG signals having less contamination with $60-\mathrm{Hz}$ noise than did those of the standard system. The lower line noise in microEEG signals was apparent in the simultaneous parallel recording from a volunteer subject with standard cup electrodes (Figures 2, 3, 4). It was also seen across 28 recordings obtained in the ED with the microEEG and the Electro-Cap compared to 18 recordings made with the standard system and cup electrodes in the ED or ICU (Figure 5).

These experiments also demonstrated high concordance between the microEEG and the standard system of spectral properties in the frequency range of physiologic EEG activity (Figures 4 and5 and Table 3, "spectral" columns with notch filter on). Not surprisingly, there was also high agreement in the time domain between the microEEG and standard system recordings when the difference in $60-\mathrm{Hz}$ noise was reduced with the notch filter (Table 2 and Table 3, "time-domain" columns, notch filter on). The fact that notch filtering the signals caused the mean levels of all measures to become nearly equal between the two systems (Table 3), combined with the data shown in Figure 3, demonstrates that the source of differences between the systems was the relatively higher line noise in the standard system. In other words, comprehensive frequency and time domain analyses of EEG signals recorded with the microEEG and standard system did not reveal any device-specific differences other than the generally higher $60 \mathrm{~Hz}$ noise in the standard system. Thus, advantages deriving from the microEEG's ease of use can be obtained without compromise by substituting the microEEG for a standard EEG machine when $\leq 26$ recording channels are needed.

The absence of systematic differences in signal properties between the microEEG and standard system is reflected in the blinded assessment of EEGs from both systems by an experienced clinical neurophysiologist. As shown in Table 4, the fraction of microEEG studies recorded in the ED with either cup electrodes or the Electro-Cap considered definitely acceptable for clinical interpretation was 58 and $60 \%$, respectively, compared to $88 \%$ for EEGs recorded with the standard system and cup electrodes in the EEG laboratory and $0 \%$ of standard system studies from the ICU. Perhaps equally important, the fraction of clinically acceptable (as well as possibly acceptable) microEEG studies in the ED did not differ significantly between those recorded with cup electrodes and the Electro-cap. In a separate prospective study of ED patients presenting with altered mental status at our institution, patients receive both a standard EEG with cup electrodes and a microEEG with the Electro-Cap. Data from the microEEG recordings in the prospective study are not yet available for analysis.

These data reveal a significant additional advantage of the microEEG - its ability to generate high-quality EEG from electrodes with high electrode-scalp impedances. Specifically, when used with the Electro-Cap, the microEEG performed well with electrode impedances substantially higher than the $5 \mathrm{k} \Omega$ recommended by professional societies, i.e., mean $15.9 \pm 17.6 \mathrm{k} \Omega$ (range 0.9 to $80 \mathrm{k} \Omega$ ) [26] and with interelectrode impedance differences within an EEG much higher than the $2 \mathrm{k} \Omega$ reported to degrade CMRR, i.e., mean $8.7 \pm 11.2 \mathrm{k} \Omega$ (range 0 to $60 \mathrm{k} \Omega$ ) [27]. This result provides a mechanism to overcome the two remaining obstacles listed above to achieving quality EEG recordings in the ED environment. In the prospective study mentioned above, the mean setup time (i.e., time between initial contact with the patient and beginning the EEG recording) was $13 \pm 7 \mathrm{~min}$ for the first 50 patients, nearly all of whom were uncooperative and many of whom were agitated. There is also no risk of an agitated patient accidentally dislodging or pulling off electrode wires after the electrodes are attached. In a later phase of this study, the microEEG and Electro-Cap hardware will be used by personnel with minimal formal training in EEG electrode placement and EEG equipment (e.g., EKG technicians) to obtain EEGs on ED patients with AMS. However, the data already obtained with EEG technologists in the ED [28], and with non-EEG technologists applying EEG electrodes in other research studies on 
healthy subjects [29], strongly suggest that the microEEG technology in combination with readily available and rapidly deployable electrode arrays will permit rapid acquisition of EEGs in the ED by personnel without the training and experience of EEG technologists. Since the $\mathrm{ED}$ is one of the most challenging environments for recording EEG, we expect that these results will be relevant for recording EEG in other challenging environments such as ICUs, as well as in the EEG laboratory.

A limitation of this study was that it used recordings obtained only from healthy adult volunteers and consequently involved no abnormal EEG patterns. This followed from the fact that it was designed as an initial pilot study to demonstrate the feasibility and safety of the microEEG as a prerequisite to a separate study of its diagnostic accuracy with patients.

\section{Conclusions}

microEEG is a miniature, wireless, battery-powered EEG device with engineering specifications equivalent to those of much larger commercially available EEG machines.

The analog EEG signals are amplified and digitized within the device, and then transmitted wirelessly to a laptop computer within 10 meters. Comprehensive time and frequency domain analyses of microEEG recordings from normal volunteers in an EEG laboratory and emergency department revealed neither unexpected signals nor significant differences from EEGs recorded with a Nicolet Monitor machine. microEEG is typically placed on or near the patient's head and can record high-quality EEG from electrodes with high electrode-scalp impedances. Its noise immunity is enhanced by the short length of electrode wires from scalp to device. This feature is particularly useful when the device is used with an electrode cap, or in a setting such as the emergency department where time and space constraints may limit a technologist's ability to achieve and maintain low electrode impedances throughout the recording.

\section{Appendix A}

The following 20 channels were used in the parallel recording: FP1, FP2, F3, F4, C3, C4, P3, P4, O1, O2, F7, F8, T3, T5, T6, FZ, CZ, PZ, LOC, and ROC. Sampling rates for the microEEG and standard system were 200 and $500 \mathrm{~Hz}$, respectively. For the recordings with the microEEG in the EEG laboratory, we used the following 16 channels: FP1, FP2, F3, F4, C3, C4, P3, P4, O1, O2, F7, F8, T3, T4, T5, and T6. The sampling rate was $250 \mathrm{~Hz}$. Signals from electrodes F7, F8, T5, and T6 were used in the analyses for the microEEG in the ED. In computing the power spectral density (PSD), the multitaper method [30] with six orthogonal tapers was used, with the size of the time series defined as the length of the FFT (Matlab's function pmtm). The spectral properties derived from EEG data were as follows: band power: the integral of the PSD over specified frequency ranges. The ranges used were delta $(0 \leq \mathrm{f}<4 \mathrm{~Hz})$, theta $(4 \leq \mathrm{f}<8 \mathrm{~Hz})$, alpha $(8 \leq \mathrm{f}<12 \mathrm{~Hz})$, beta1 $(12 \leq \mathrm{f}<14 \mathrm{~Hz})$, beta2 $(14 \leq \mathrm{f}<16 \mathrm{~Hz})$, beta3 $(16 \leq \mathrm{f}<20 \mathrm{~Hz})$, beta4 $(20 \leq \mathrm{f}<24 \mathrm{~Hz})$, beta5 $(24 \leq \mathrm{f}<32 \mathrm{~Hz})$, high frequency $(32 \leq \mathrm{f}<70 \mathrm{~Hz})$, and power line noise $(59 \leq \mathrm{f}<61 \mathrm{~Hz})$. Band power can be considered as a frequency-specific contribution to the overall variance of the signal. Mean frequency: the first moment of the normalized PSD. SEF75: The $75 \%$ spectral edge frequency, that is, the frequency that contains $75 \%$ of all the power in the spectrum (starting at $\mathrm{f}=0 \mathrm{~Hz}$ ). Spectral entropy: the entropy of the normalized PSD interpreted as a discrete probability distribution. This ranges between 0 for a maximally concentrated PSD tothe value $\log _{2}(\mathrm{~N})$, for a uniform distribution, where $\mathrm{N}$ is the total number of discrete frequencies in the PSD ( $\mathrm{N}$ was constant for all channels and for both systems).

\section{Competing interests}

Supported by NIH grant 1RC3NS070658 to Bio-Signal Group (BSG), with a subcontract to SUNY Downstate Medical Center. André Fenton is a founder of BSG. Samah Abdel-Baki and Ahmet Omurtag are employees of Bio-Signal Group and with André Fenton are co-inventors listed on a patent application based on the microEEG (pub. no. WO/2010/129026. Title: EEG Kit). ACG serves on the BSG advisory board. All income derived from this position is donated directly from BSG to the Downstate College of Medicine Foundation.

\section{Authors' contributions}

Drs. Abdel Baki and Omurtag contributed equally to this paper. All authors read and approved the final manuscript.

\section{Acknowledgements}

The authors would like to thank Dr. Shweta Malhotra for her invaluable assistance in data collection and Dr. Richard Sinert for generously making ED resources available for this study.

\section{Author details}

'Bio-Signal Group, 760 Parkside Avenue, Ste 206, Brooklyn, NY 11226, USA

${ }^{2}$ Departments of Neurology and Pediatrics, State University of New York, Downstate Medical Center, Brooklyn, USA. ${ }^{3}$ Department of Neurology, State University of New York, Downstate Medical Center, Brooklyn, USA.

${ }^{4}$ Department of Emergency Medicine, State University of New York, Downstate Medical Center, Brooklyn, USA. ${ }^{5}$ Center for Neural Science, New York University, New York and Department Physiology \& Pharmacology, State University of New York, Downstate Medical Center, Brooklyn, USA. ${ }^{6}$ Departments of Neurology, and Physiology \& Pharmacology, State University of New York, Downstate Medical Center, Brooklyn, USA.

Received: 9 April 2012 Accepted: 31 August 2012

Published: 24 September 2012

\section{References}

1. Kanich W, Brady WJ, Huff JS, Perron AD, Holstege C, Lindbeck G, et al: Altered mental status: evaluation and etiology in the ED. Am J Emerg Med 2002, 20:613-617.

2. Riggio S: Psychiatric manifestations of nonconvulsive status epilepticus. Mt Sinai J Med 2006, 73:960-966. 
3. Ziai WC, Schlattman D, Llinas R, Venkatesha S, Truesdale M, Schevchenko A, Kaplan PW: Emergent EEG in the emergency department in patients with altered mental states. ClinNeurophysiol 2012, 123(5):910-917.

4. Saengpattrachai M, Sharma R, Hunjan A, Shroff M, Ochi A, Otsubo H, Cortez MA, Carter Snead O: Nonconvulsive Seizures in the Pediatric Intensive Care Unit. Epilepsia 2006, 47(9):1510-1518.

5. Bautista RE, Godwin S, Caro D: Incorporating abbreviated EEGs in the initial workup of patients who present to the ER with mental status changes of unknown etiology. J ClinNeurophysiol 2007, 24(1):16-21.

6. Moeller JJ, Kurniawan J, Gubitz GJ, Ross JA, Bhan V: Diagnostic accuracy of neurological problems in the emergency department. Can J NeurolSci 2008, 35:335-341.

7. Moulin T, Sablot D, Vidry E, Belahsen F, Berger E, Lemounaud P, Tatu L, Vuillier F, Cochon A, Revenco E, Capellier G, Rumbach L: Impact of emergency room neurologists on patient management and outcome. EurNeurol 2003, 50:207-214

8. Roberts K, Costelloe D, Hutchinson M, Tubridy N: What difference does a neurologist make in a general hospital?/r. J Med Sci 2007, 176(3):211-214.

9. Abdel Baki SG, Omurtag A, Fenton AA, Zehtabchi S: The new wave: time to bring EEG to the emergency department. IntJEmerg Med 2011, 4:36-43.

10. Kaplan PW: Assessing the outcomes in patients with NCSE: nonconvulsive status epilepticus is underdiagnosed, potentially overtreated, and confounded by comorbidity. J ClinNeur 1999, 16(4):341-342

11. Drislane FW, Blum AS, Schomer DL: Unsuspected electrographic status epilepticus in intensive care units. Neurology 1998, 50(suppl 1):A395-A396.

12. Bastani A, Kayyali H, Schmidt R, Qadir R, Manthena P: Wireless brain monitoring in the emergency department. ConfProc IEEE Eng Med BiolsoC 2005, 3:2502-2505

13. Hjorth B: EEG analysis based on time domain properties. ElectroencephalogrClinNeurophysiol 1970, 29:306-310.

14. Drongelen WV: Signal Processing for Neuroscientists: An Introduction to the Analysis of Physiological Signals. Burlington MA, USA: Academic Press/ Elsevier; 2006:68

15. Sanei S, Chambers JA: EEG Signal Processing. Wiley, New York, NY, USA: Wiley-Interscience; 2007:51.

16. Faul S, Boylan G, Connolly S, Marnane L, Lightbody G: An evaluation of automated neonatal seizure detection methods. ClinNeurophysiol 2005, 116:1533-1541.

17. Meier R, Dittrich H, Schulze-Bonhage A, Aertsen A: Detecting epileptic seizures in long-term human EEG: anew approach to automatic online and real-time detection and classification of polymorphic seizure patterns. J ClinNeurophys 2008, 25:119-131.

18. Delorme A, Sejnowski T, Makeig S: Enhanced detection of artifacts in EEG data using higher order statistics and independent component analysis. Neuroimage 2007, 34:1443-1449.

19. Mormann F, Adrzejak RG, Elger CR, Lehnertz K: Seizure prediction: the long and winding road. Brain 2007, 130:314-333.

20. Minasyan GR, Chatten J, Chatten M, Harner RN: Patient-specific early seizure detection from scalp electroencephalogram. J ClinNeurophys 2010, 27:163-178.

21. Fell J, Roeschke J, Mann K, Schaeffner C: Discrimination of sleep stages: a comparison between spectral and nonlinear EEG measures. ElectroencephalogrClinNeurophysiol 1996, 98:401-410.

22. Ferri $\mathrm{R}$, Cosentino $\mathrm{Fl}$, Elia M, Musumeci SA, Marinig R, Bergonzi $\mathrm{P}$ : Relationship between delta, sigma, beta, and gamma EEG bands at REM sleep onset and REM sleep. Clinical Neurophsiol 2001, 112:2046-2052.

23. Mantini D, Perucci MG, Gratta CD, Romani GL, Corbetta GLM: Electrophysiological signatures of resting state networks in the human brain. PNAS 2007, 104:13170-13175.

24. Wendling F, Bartolomei F, Senhadji L: Spatial analysis of intracerebral electroencephalographic signals in the time and frequency domain: identification of epileptogenic networks in partial epilepsy. Philos Transact A Math PhysEngSci 2009, 367(Wendling F):297-316.

25. Press W, Teukolsky S, Vetterling W, Flannery B: Numerical Recipes. New York, NY, USA: Cambridge University Press; 2007:120-124.

26. Nuwer MR, Comi G, Emerson R, Fuglsang-Frederiksen A, Guerit JM, Hinrichs $H_{\text {, }}$ Ikeda A, Luccas FJ, Rappelsburger P: Int Fed ClinNeurophysiol. ElectroencephalogrClinNeurophysiol 1998, 106:259-261.
27. Legatt A: Impairment of common mode rejection by mismatched electrode impedances: quantitative analysis. Am J EEG Technol 1995, 35:296-302.

28. Zehtabchi S, Grant AC, Abdel Baki SG, Omurtag A, Sinert R, Chari G, et al: Diagnostic accuracy of a novel emergency electroencephalography device (microEEG) in identifying non-convulsive seizures and other EEG abnormalities in the emergency department patients with altered mental status. AcadEmerg Med 2012, 19(S1):s378.

29. Kolls BJ, Olson DM, Gallentine WB, Skeen MB, Skidmore CT, Sinha SR: Electroencephalography leads placed by nontechnologists using a template system produce signals equal in quality to technologistapplied, collodion disk leads. J ClinNeurophysiol 2012, 29(1):42-49.

30. Percival DB, Walden A: Spectral Analysis for Physical Applications: Multitaper and Conventional Univariate Techniques. New York, NY, USA: Cambridge University Press; 1998:331-375.

doi:10.1186/1865-1380-5-35

Cite this article as: Omurtag et al:: Technical and clinical analysis of microEEG: a miniature wireless EEG device designed to record highquality EEG in the emergency department. International Journal of Emergency Medicine 2012 5:35.

\section{Submit your manuscript to a SpringerOpen ${ }^{\odot}$ journal and benefit from:}

- Convenient online submission

- Rigorous peer review

- Immediate publication on acceptance

- Open access: articles freely available online

- High visibility within the field

- Retaining the copyright to your article

Submit your next manuscript at $>$ springeropen.com 